\title{
Remineralization of dentin induced by a compound of polyamide-amine and chlorhexidine in a resin dentin bonding microenvironment
}

\author{
Kezhen Xiang, Liang Chen, Wang Chen, Deqin Yang \\ Department of Endodontics, Stomatological Hospital Affiliated to Chongqing Medical University, Chongqing, China \\ Contributions: (I) Conception and design: L Chen; (II) Administrative support: D Yang; (III) Provision of study materials or patients: W Chen; (IV) \\ Collection and assembly of data: K Xiang; (V) Data analysis and interpretation: K Xiang; (VI) Manuscript writing: All authors; (VII) Final approval of \\ manuscript: All authors. \\ Correspondence to: Deqin Yang. Department of Endodontics, Stomatological Hospital Affiliated to Chongqing Medical University, Chongqing 401120, \\ China. Email: yangdeqin@hospital.cqmu.edu.cn.
}

Background: The purpose of this study was to investigate the effect of a complex of polyamide-amine dendrimer (PAMAM) and chlorhexidine gluconate (CG) on remineralization of dentin in an artificial simulated resin dentin bonding microenvironment.

Methods: The structure of this complex was characterized by FT-IR. Twelve standard dentin samples were randomly divided into four treatment fluid groups namely a PAMAM group, CG group, PAMAM + CG group, and deionized water group. A microenvironmental mineralization model was established in vitro with $50 \mu \mathrm{m}$ gap width between resin and dentin. The dentin surface was observed by a scanning electron microscope (SEM), and the chemical structure of the surface was analyzed by X-ray energy spectrum (EDS), $\mathrm{X}$-ray diffraction (XRD), and laser Raman spectroscopy.

Results: SEM showed the density of dentinal tubules exposed in the PAMAM group decreased after a 14-day immersion, with corn rod-shaped crystal structures gathered around the tubules. In addition, visible mineralization occurred in partial areas of the CG group, rod-shaped crystals and in comparison, dentinal tubules in the PAMAM + CG group were almost completely covered by flaky crystal structures. Raman spectrum analysis showed that crystals formed by PAMAM, CG, and PAMAM + CG solution all had strong phosphate characteristic peaks, indicating the presence of hydroxyapatite (HA), that of the PAMAM + CG group was the strongest. The EDS results showed that the Ca and P levels of the PAMAM group and the CG group were slightly higher than those of the deionized water group, while PAMAM + CG group significantly higher than the others, $\mathrm{Ca} / \mathrm{P}$ value approaching 1.67. The results of XRD showed the characteristic peaks of hydroxyapatite detected by the PAMAM + CG group at 2 $\theta=26.0$ (002), $2 \theta=32.0$ (211), and $2 \theta=33.0$ (112) were high and sharp, with a few diffraction line burrs indicating it had high crystallinity and purity. The Scherrer equation results showed that the appearance and size of the grains formed by the PAMAM + CG group were basically consistent with those of healthy dentin.

Conclusions: Altogether, the compound of polyamide-amine dendrimer and chlorhexidine could induce the remineralization of human dentin in a resin dentin bonding microenvironment with a gap of $50 \mu \mathrm{m}$ to form a crystal structure similar to dentin hydroxyapatite.

Keywords: Polyamide-amine; chlorhexidine; remineralization; micro-leakage environment; dentin apatite

Submitted Dec 24, 2020. Accepted for publication Mar 16, 2021.

doi: $10.21037 / \mathrm{atm}-21-472$

View this article at: http://dx.doi.org/10.21037/atm-21-472 


\section{Introduction}

Unlike bone tissue, human dentin cannot self-repair after being damaged due to a lack of cellular components. At present, the most common clinical treatment method to repair dentin defects involves the use of composite resin materials for bonding and filling. However, clinical and laboratory results have confirmed that the long-term effect of resin dentin bonding is unsatisfactory and eventually leads to problems such as restoration detachment and secondary caries (1-3). The resin dentin bonding interface is the weak environment in resin repair technology, and there are two key problems in this interface: the formation of nano-microleakage and the degradation of collagen fibers by matrix metalloproteinase (MMP). Research on nanomicroleakage at the bonding interface mainly focuses on how to promote remineralization of dentin and closing the microleakage gap by newly formed crystals $(4,5)$. Three basic conditions must be present for remineralization: Firstly, there must be a complete collagen fiber structure as a scaffold for the growth of mineral crystals $(6,7)$; secondly, there should be residual mineral crystals as growth centers, or, at least in the case of complete demineralization, there should be newly formed nucleation centers (8); and thirdly, a mineral source containing calcium and phosphorus must be provided (9). To achieve these conditions, many scholars have devoted themselves to studying new mineralized materials, such as fluoride, polyaspartic acid, and polyamideamine dendrimer $(10,11)$. Research on the degradation of collagen fibers by matrix metalloproteinases (MMPs) has largely involved adding MMPs inhibitors to the bonding process, such as chlorhexidine (CHX), Gluma and natural extracts $(12,13)$, which can inhibit the activity of MMPs in dentin and prevent the degradation of collagen fibers, thus enhancing the long-term effect of interfacial bonding.

Some scholars have also studied the application of mineralized materials in combination with other materials $(14,15)$. For example, two antibacterial agents (NAG, DMAHDM) and one remineralization agent (NACP) have been used in combination to simultaneously produce an antibacterial/remineralization function and reduce the carcinogenicity of biofilm, and improve the strength and fatigue resistance of the dentin-resin bonding interface (16). However, there are no reports on the combined application of mineralized materials and MMPs inhibitors.

Accordingly, although many studies have achieved good results in nano-microleakage or collagen fiber degradation, there are still limitations, which does not allow for consideration to both problems at the same time.

Chlorhexidine is a strong alkali with cationic properties after being ionized in water and has four main advantages. As a common protease inhibitor, it can obviously inhibit the activity of matrix metalloproteinases (MMP-2, -8 and -9) (17); it has a strong affinity with tooth tissue (18); it has a strong bacteriostatic effect which can reduce the acid resistance of Streptococcus mutans, and it can reduce the rate of environmental $\mathrm{PH}$ reduction (19). Some research shows that $0.2-2 \%$ CG can promote the interfiber remineralization of incompletely demineralized dentin and produce granular crystals in a short time (20), but the mineralization effect is limited. The protective effect of chlorhexidine molecules on collagen fibers often begins to decline obviously after $12-14$ months due to the washing of dentin canal fluid and limited binding force (21). Therefore, the author of this experiment proposed that the remineralization rate of demineralized dentin should be increased as much as possible, that remineralization should be completed before the elution failure of chlorhexidine molecules, and that the crystal structure induced by chlorhexidine mineralization still needs to be analyzed by Raman and XRD. In recent years, PAMAM dendrimers have become the research focus of biomimetic mineralization. Several studies have shown that PAMAM with different end groups can be used as good remineralizers to induce remineralization of partially demineralized dentin $(22,23)$ and can even be used as the mineralization center on the surface of completely demineralized dentin to remineralize collagen fibers. This may be because PAMAM can induce and regulate the growth of mineralized crystals and can be regarded as a biomimetic mimic of dentin non-collagen (NCPs), which can promote the appearance and composition of new crystals to be close to natural hydroxyapatite $(24,25)$.

Inspired by the above research, we sought to solve the problems of nano-microleakage and matrix metalloproteinases (MMPs) degradation of collagen fibers at the same time and improve the long-term adhesion between resin and dentin. To do so, we synthesized a PAMAM$\mathrm{COOH}+\mathrm{CG}$ composite and studied its remineralization ability in the $50 \mu \mathrm{m}$ interstitial microenvironment simulating microleakage, to provide a preliminary basis for the subsequent study of multiple functions of the composite. The morphology of remineralized dentin was characterized by scanning electron microscope (SEM), and the mineralized crystals were qualitatively and quantitatively analyzed by energy dispersive X-ray (EDX), 
Raman spectroscopy, and X-ray diffractometer (XRD). We assumed that the PAMAM-COOH + CG complex could induce remineralization of demineralized dentin, and that the regenerated crystals are similar to natural dentin.

We present the following article in accordance with the MDAR reporting checklist (available at http://dx.doi. org/10.21037/atm-21-472).

\section{Methods}

The following materials were used: Fresh caries-free third molars, 35\% phosphate gel (Gluma, Germany), ALLBOND self-etching adhesive (Bisco, USA), Z350 lightcured composite resin (3M, USA), artificial saliva (Solebao, Beijing), G4-PAMAM-COOH (Weihai Chenyuan Company, Shandong), $2 \%$ chlorhexidine gluconate, Bluephase N MC UV-curing lamp (Hikviewadent, Liechtenstein), hard tissue microtome (EXAKT, Germany), field emission scanning electron microscope and energy spectrum analyzer (Hitachi, Nippon), Fourier transform infrared spectrometer (Nicolet, USA), laser Raman spectrometer (LabRAM HR, Germany), and X-ray diffractometer (Shimadzu, Japan).

\section{Sample preparation of dentin sheet}

Twelve recently extracted human third molars were selected after the informed consent of donors was obtained. The specimens were collected according to the guidelines of the Medical Ethics Committee of the Chongqing Medical University. All procedures performed in this study involving human participants were in accordance with the Declaration of Helsinki (as revised in 2013). The study was approved by the Ethics Committee of Stomatological Hospital Affiliated to Chongqing Medical University (No. 2019-75) and informed consent was taken from all the patients. The teeth were required to be free of stains, uniform in color, non-tetracycline or dental fluorosis, and alive teeth before tooth extraction. The age of patients ranged from 20 to 50 years and the specimens had been stored in $0.02 \%$ thymol at $4{ }^{\circ} \mathrm{C}$. A dentin slice with a thickness of $1 \mathrm{~mm}$ was cut with a low-speed saw at the position $1.5 \mathrm{~mm}$ above the enamel dentin boundary in the direction perpendicular to the long axis of each tooth. Twelve dentin slices were then prepared, and the observation surface of slices was wet polished with 320 -grit then 600 -grit silicon carbide sandpaper for $30 \mathrm{~s}$, respectively. The observation surface was etched with $35 \%$ phosphoric acid gel for $60 \mathrm{~s}$ and rinsed with deionized water for $60 \mathrm{~s}$ to form completely demineralized dentin with a thickness of about 5-8 $\mu \mathrm{m}$ (26), then ultrasonically vibrated for $15 \mathrm{~min}$ at $150 \mathrm{~W}$.

\section{Preparation and characterization of PAMAM-COOH + CG complex}

This was performed by adding $10 \mathrm{mg} / \mathrm{mL}$ PAMAM$\mathrm{COOH}$ solution into $2 \% \mathrm{CG}$ solution, mixing, then placing the solution in a water bath at $37^{\circ} \mathrm{C}$ for $1 \mathrm{~h}$, followed by shaking in a shaker at $37^{\circ} \mathrm{C}$ for $24 \mathrm{~h}$, separating with a $3 \mathrm{kD}$ ultrafiltration tube, and centrifuging at 4,000 rpm for $1 \mathrm{~h}$. The supernatant was then freeze-dried to obtain the PAMAM + CG complex and separated with a $3 \mathrm{kD}$ ultrafiltration tube.

\section{Treatment of dentin samples}

The above samples were randomly divided into four groups (n=3); a PAMAM group, CG group, PAMAM + CG group, and deionized water group. The demineralized dentin surface of each group was soaked in $10 \mathrm{mg} / \mathrm{mL}$ G4PAMAM-COOH (PAMAM group), 2\% CG, G4-PAMAM$\mathrm{COOH}(10 \mathrm{mg} / \mathrm{mL})+2 \%$ CG (PAMAM + CG group), and deionized water (deionized water group) for $1 \mathrm{~h}$, then rinsed with deionized water for $5 \mathrm{~s}$.

A Z350 light-cured composite resin (3M, USA) was placed between two glass plates, and a light-cured composite resin block with a thickness of about $1 \mathrm{~mm}$ was laminated, which was cured by illumination for $20 \mathrm{~s}$, and the output intensity was $800 \mathrm{~mW} / \mathrm{cm}^{2}$. Sandpaper of 320 and 600 meshes were used to polish the surface and acid etching was used for $15 \mathrm{~s}$ to remove impurities. The resin was then washed with deionized water for $15 \mathrm{~s}$ then air dried.

A transparent molding sheet with a thickness of $50 \mu \mathrm{m}$ was placed between the observation surface of the prepared dentin sample and the resin block, and both ends were fixed with self-etching adhesive and flowing resin and cured by illumination as described above. The molding sheet was then drawn out, and a resin dentin bonding microenvironment model with a gap width of $50 \mu \mathrm{m}$ was made (Figure 1). Samples of the microenvironment model were stored in artificial saliva $(\mathrm{pH}=7.0)$ at $37^{\circ} \mathrm{C}$, and the artificial saliva was changed every $24 \mathrm{~h}$, before being taken out for detection and analysis on the 14th day.

The Z350 resin composite coated with self-etching adhesive at both ends was placed above the measuring surface of human dentin (the dentin block was $1 \mathrm{~mm}$ thick), 


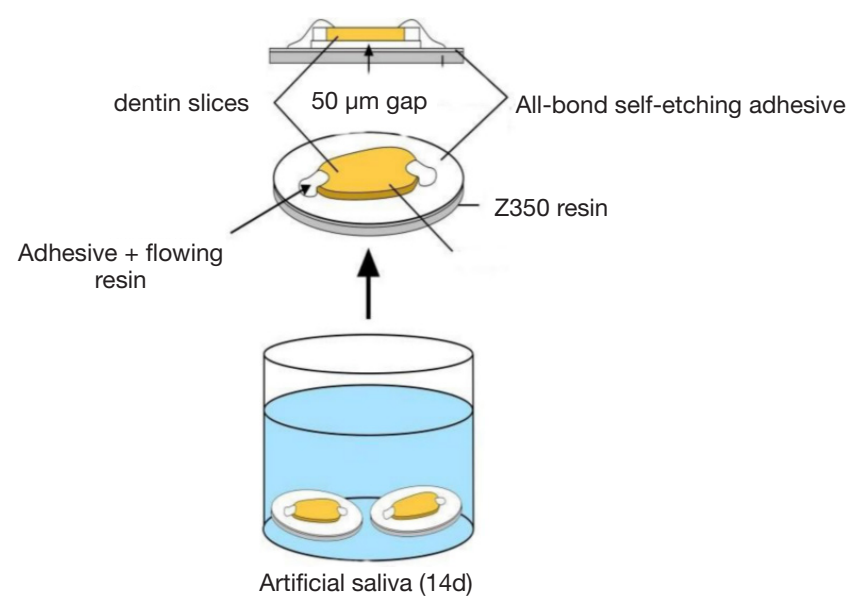

Figure 1 Schematic diagram of experimental process.

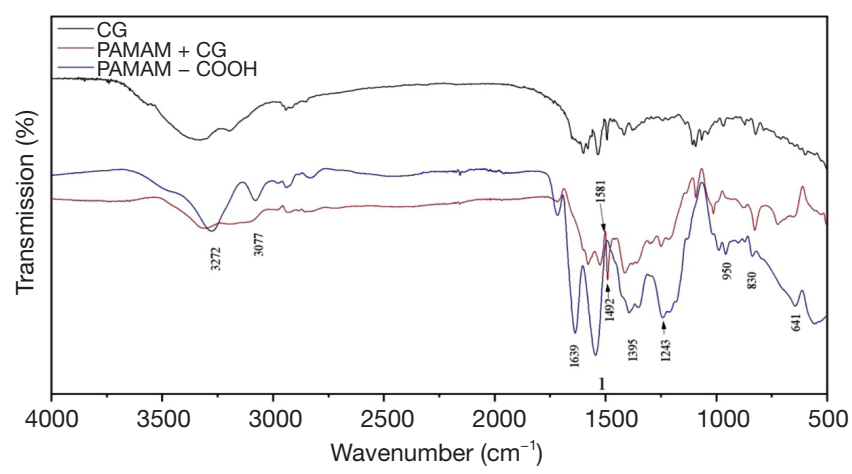

Figure 2 FT-IR spectra of PAMAM-COOH and CG complex. PAMAM, polyamide-amine dendrimer; CG, chlorhexidine gluconate.

the middle was separated by a transparent molding sheet with a thickness of $50 \mu \mathrm{m}$, and the two ends were fixed by light curing with flowing resin, thus forming a resin dentin bonding microenvironment model with a gap of $50 \mu \mathrm{m}$.

\section{SEM and EDX detection}

The resin block and dentin block were separated from the bonding point, and the dentin block was then dehydrated by ethanol gradient. After being fixed by glutaraldehyde, this was freeze-dried and sprayed with gold and mineralization of the dentin surface was observed by SEM under the condition of $5 \mathrm{kV}$. The elements of calcium (Ca), phosphorus $(\mathrm{P})$, carbon $(\mathrm{C})$, and oxygen $(\mathrm{O})$ were analyzed by EDX under the condition of $20 \mathrm{kV}$. Four test points were taken for each sample.

\section{Laser Raman spectroscopy}

The changes of surface phosphate $\mathrm{PO}_{4}{ }^{3-}$ peak intensity of four groups of demineralized dentin soaked for 14 days were detected by laser Raman spectroscopy. The wavelength range of the Raman spectrum was $900-1,700 \mathrm{~cm}^{-1}$, $600^{-}$lines $/ \mathrm{mm}$, and the resolution was $4 \mathrm{~cm}^{-1}$. The spectral data were analyzed by OMNIC 8 software, the background was removed, the baseline was corrected, and the changes of phosphate peak intensity in each group were compared.

\section{$X R D$}

An XRD diffractometer was used to detect crystal formation on the surface of four groups of completely demineralized dentin after soaking for 14 days. The testing parameters of XRD diffractometer were as follows: $\mathrm{Cu}-\mathrm{k} \alpha$ ray, voltage $40 \mathrm{kV}$, current $40 \mathrm{~mA}$, Ni filter, scanning angle 25-65. The XRD data were analyzed by MDI Jade 9 software. The XRD patterns obtained were compared with the Joint Committee on Power Diffraction (JCPDS), and the diffraction peak intensity ratios along the $\mathrm{c}$ and a axes and the average grain sizes in the two directions were calculated according to the indexed XRD peaks of (002) and (211), using the Scherrer equation (25) as follows:

$\mathrm{D}=\mathrm{K} \lambda / \beta \cos \theta(\mathrm{nm})$

$\mathrm{D}$ is the grain size in nanometers, $\mathrm{k}$ is the Scherrer constant ( $\mathrm{K}=0.9), \lambda$ is the $\mathrm{X}$-ray wavelength in nanometer $(\lambda=0.154), \beta$ is the half maximum intensity of diffraction peak, and $\theta$ is the diffraction angle of the diffraction peak. The data obtained from each group were compared with healthy dentin without demineralization, and the phase composition and crystal structure of the samples were analyzed.

\section{Results}

\section{FT-IR analysis}

We used FT-IR analysis to determine whether CG molecules were loaded on PAMAM-COOH macromolecules, and the results are shown in Figure 2. Compared with PAMAM-COOH, the PAMAM + CG complex not only shows the characteristic peak of PAMAM-COOH at $1,243 \mathrm{~cm}^{-1}$ but also shows the vibration absorption peaks of the benzene ring skeleton of CG drug molecules at 1,581, 1,492 $\mathrm{cm}^{-1}$ and other places (as shown by the arrows). 


\section{SEM and EDX results}

SEM observation: Compared with the deionized water group (Figure $3 A, B$ ), the dentin tubes per unit area in the PAMAM group were sparse after 14 days of complete demineralization, and many corn rod-like crystal structures were gathered around the tubes, leaving only a small amount of fiber gap. In addition, the crystals were deposited radially along collagen fibers in dentinal tubules, which almost completely blocked the tubules (Figure $3 C, D$ ). In the deionized water group, a small amount of sediment adhered to the dentin surface, and the dentinal tubules remained completely open. After 14 days of complete demineralization of dentin in the CG group, visible mineralization occurred in some areas, and several rod-shaped crystals with a diameter of approximately 150-200 nm grew radially along the dentin surface, completely covering the dentin orifice (Figure $3 E, F$ ). In other areas, dentin tubes were seen to be sparse under electron microscopy, and spherical crystals were attached to the surface of demineralized collagen fibers. Although the network structure of collagen fibers can still be observed in the tubules, it is not obvious in the inter-tubule area (Figure $3 G, H$ ). After 14 days of complete demineralization of dentin in the PAMAM + CG group, the dentin blocks could be seen to be mineralized with the naked eye, and many dense lamellar mineralized structures were arranged along the collagen fibers around the tubules. Microscopically, the dentinal tubules were completely covered (Figure 3I,7). EDX results showed that the newly formed mineral layers on the surface of completely demineralized dentin were rich in $\mathrm{Ca}$ and $\mathrm{P}$ after remineralization of PAMAM, CG, and $\mathrm{PAMAM}+\mathrm{CG}$, and the $\mathrm{Ca} / \mathrm{P}$ ratios were $1.94,1.79$, and 1.87 , respectively, which is close to natural hydroxyapatite $(\mathrm{Ca} / \mathrm{P}$ is 1.67$)$. In the deionized water group, the $\mathrm{Ca} / \mathrm{P}$ ratio was 2.04, and the contents of $\mathrm{Ca}$ and $\mathrm{P}$ on the dentin surface were lower (Figure 4). The results of surface scanning (Figure 5) showed that the contents of $\mathrm{Ca}$ and $\mathrm{P}$ on the surface of mineralized dentin in the PAMAM + CG group were significantly higher than those in the other three groups, and the contents of $\mathrm{C}$ and $\mathrm{O}$ on the surface of the PAMAM + CG group and Pamam group were higher than those in CG group and deionized water group.

\section{Analysis result of Raman spectrum}

As shown in Figure 6, the phosphate peak at $960 \mathrm{~cm}^{-1}$ wavelength represents hydroxyapatite, the phosphate peak at $1,030 \mathrm{~cm}^{-1}$ wavelength represents the phosphate peak in stoichiometric hydroxyapatite (i.e., the best ratio), and the amide III peak is represented in the wavelength range of $1,050-1,430 \mathrm{~cm}^{-1}$. On the premise of removing background interference, the characteristic peak of amide bond -CO$\mathrm{NH}$-(Amide) appeared at $1,330 \mathrm{~cm}^{-1}$ in the deionized water group, which was the characteristic peak of type III collagen fiber. The other three experimental groups all showed strong phosphate characteristic peaks (hydroxyapatite characteristic peaks), among which the PAMAM + CG group had the strongest phosphate characteristic peak at $1,030 \mathrm{~cm}^{-1}$.

\section{$X R D$}

XRD analysis results of the experimental group and deionized water group samples are shown in Figure 7. The normal dentin phase is mainly composed of hydroxyapatite. Peak shapes of dentin surface products in the PAMAM + CG group are sharp and clear, and obvious diffraction peaks appear when the diffraction angles $2 \theta$ are 26 and 31.8. The positions of these characteristic peaks correspond to the standard card of hydroxyapatite diffraction pattern provided by the International Centre for Diffraction Data (JCPDS) and are basically consistent with the diffraction peaks of healthy dentin. The diffraction peak of the deionized water group was weak after remineralization for 14 days.

The peak intensity ratio of the c-axis (002) and (211) represents the diffraction intensity ratio (Table 1). The peak intensity ratio of each group was close to that of healthy dentin, and the diffraction intensity ratio of PAMAM group was slightly higher. The average grain size of the PAMAM + CG group in the c-axis direction was much larger than that of other groups including healthy dentin, and the aspect ratio of grains in the c-axis and a-axis direction was larger than that of the PAMAM group, CG group, and deionized water group, which is closest to healthy dentin.

\section{Conclusions}

The formation of human dental hard tissue is a typical biomineralization process which involves the nucleation, growth, aggregation, and orientation of crystals induced by organic matrix template $(27,28)$. Based on this "organic matrix regulation theory", a gradual recognition that organic macromolecules are the core of biomineralization and biomimetic synthesis, and molecular biomimetic design of biomaterials has become the leading premise guiding modern hard tissue repair material design. 

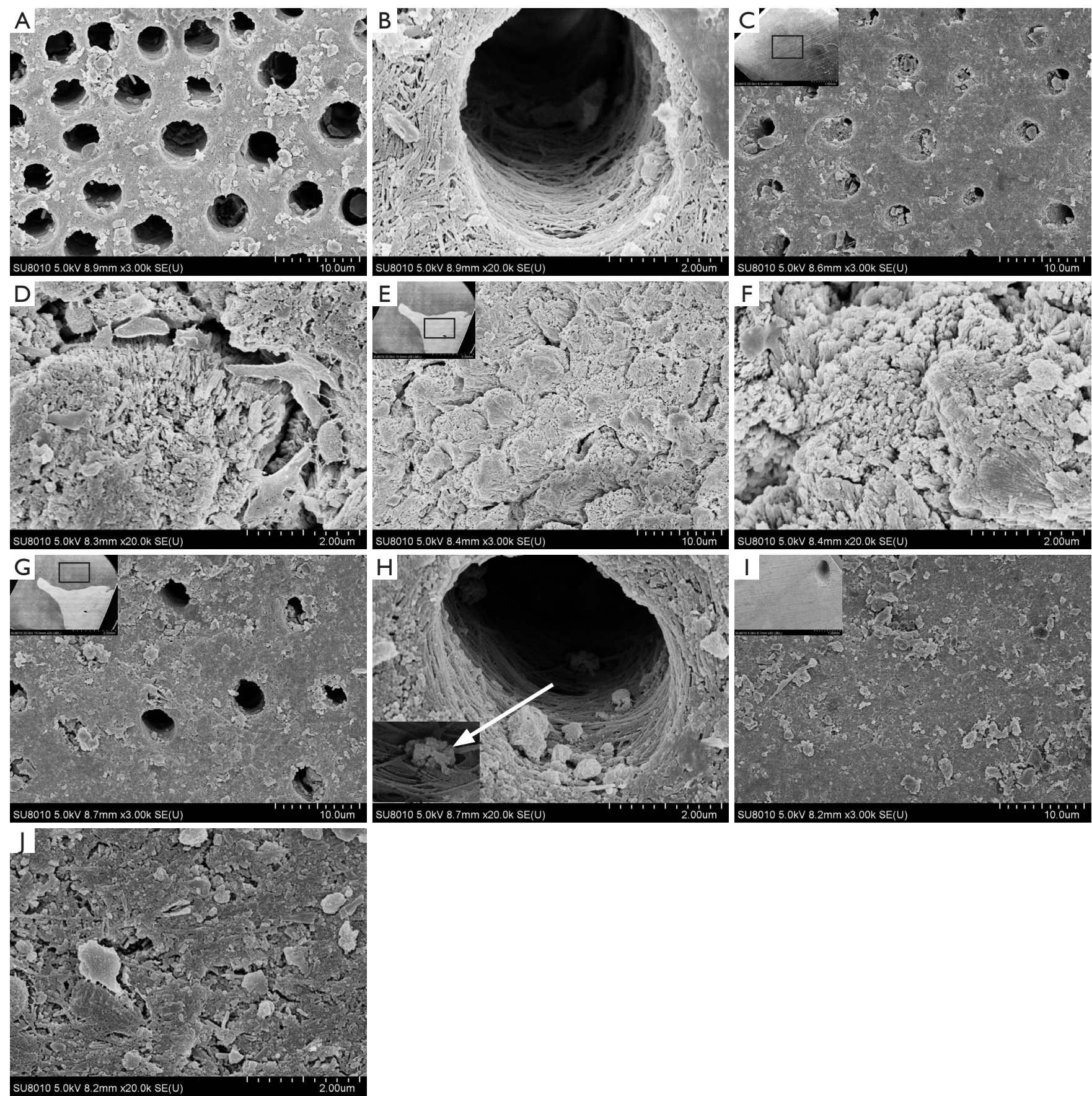

Figure 3 Scanning electron microscope (SEM) was used to observe the morphological changes of dentin surface after different treatments for 14 days. (A) Deionized water group $(\times 3,000)$; (B) deionized water group $(\times 20,000)$; (C) PAMAM-COOH group ( $\times 3,000)$; (D) PAMAMCOOH group (×20,000); (E,G) CG group (×3,000); (F,H) CG group (×20,000); (I) PAMAM-COOH + CG group $(\times 3,000)$; (J) PAMAM$\mathrm{COOH}+\mathrm{CG}$ group $(\times 20,000)$. PAMAM, polyamide-amine dendrimer; CG, chlorhexidine gluconate. The small picture in the upper left corner of A2-A5 shows the appearance of the sample under 30x electron microscope. The small picture in the lower left corner of B4 shows the enlarged appearance of the dentin tube, and the arrow marks the remineralization center composed of crystals. 

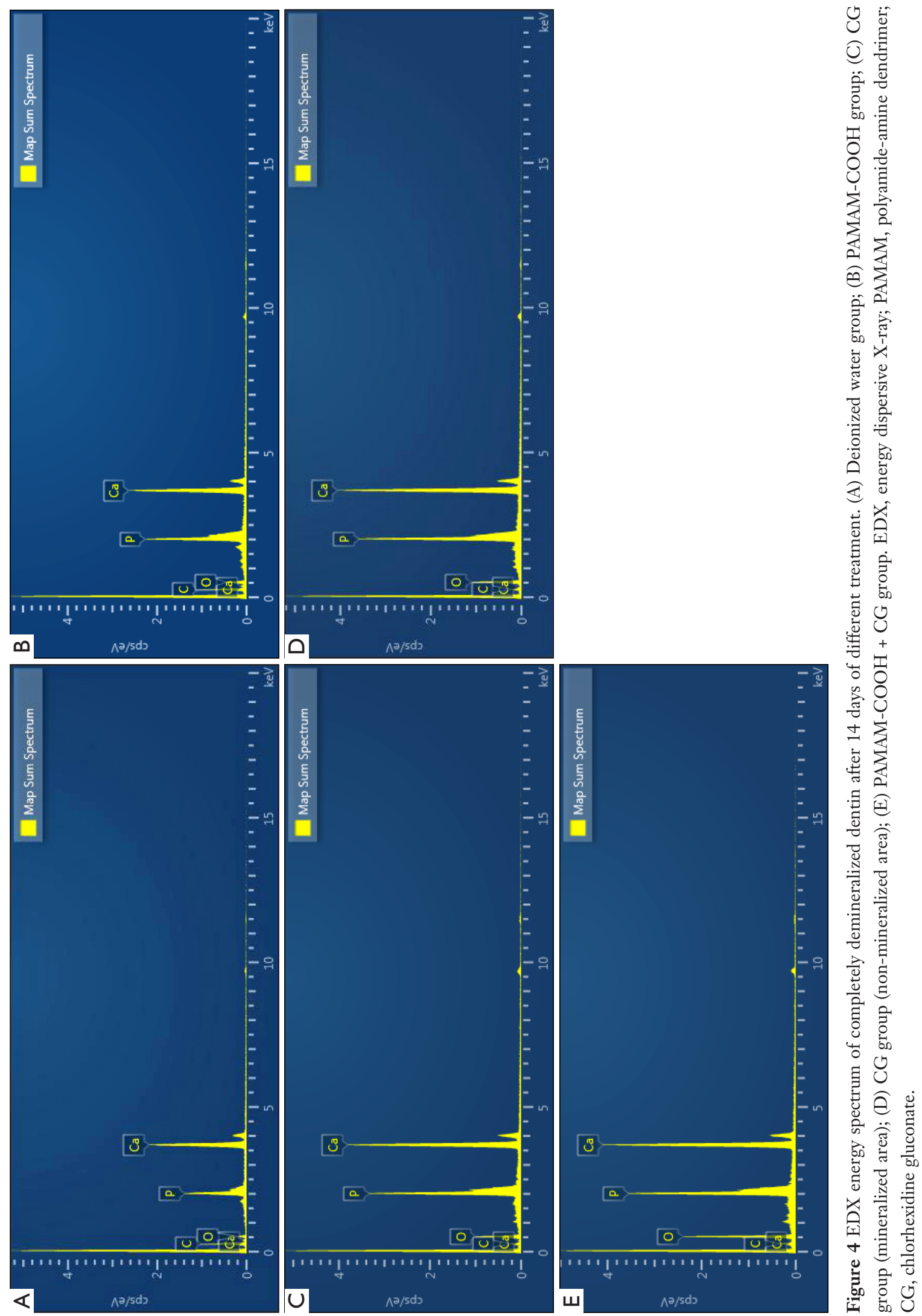

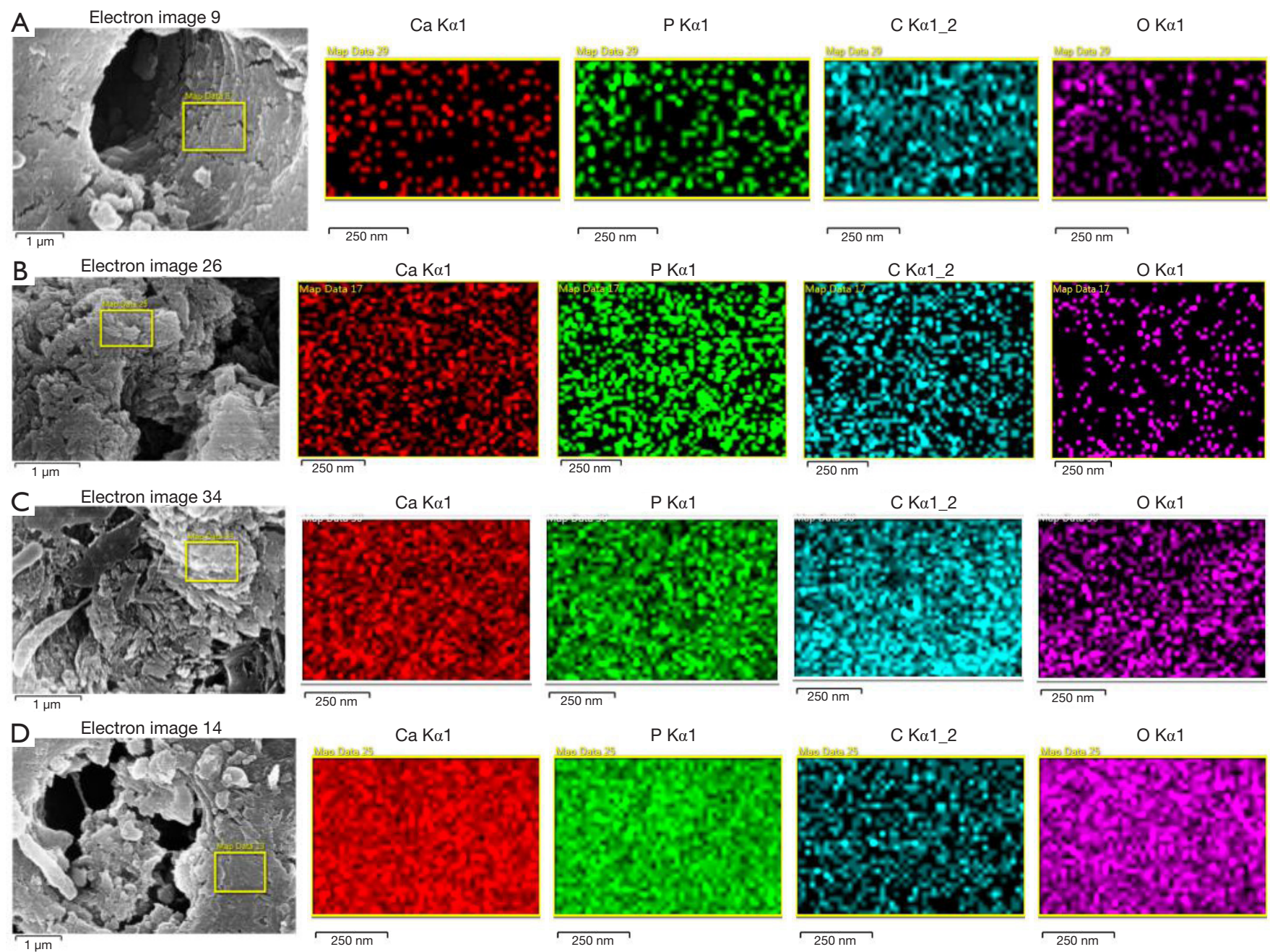

Figure 5 EDX scanning results of completely demineralized dentin after 14 days of different treatments. (A) Deionized water group; (B) PAMAM-COOH group; (C) CG group; (D) PAMAM-COOH + CG group. EDX, energy dispersive X-ray; PAMAM, polyamide-amine dendrimer; CG, chlorhexidine gluconate.

In this experiment, the remineralization effect of a compound of polyamide-amine dendrimer and chlorhexidine on dentin surface was studied. The polyamide-amine polymer has become a research hotspot because of its superior biomineralization ability $(4,5)$ and a characteristic of PAMAM is that it has internal cavities and many reactive terminal groups. These structures enable PAMAM to simulate the biomimetic mineralization of natural organic matrix on the tooth tissue surface, allowing PAMAM to be used as an organic template to control mineral nucleation and crystal growth to build a more ideal dental restoration material than traditional allogenic materials. The main process of biomimetic mineralization of dental tissue induced by an organic template sees organic self-assembly formed as the mineralization template. This is followed by inorganic precursors reacting chemically at the interface between the self-assembly aggregate and solution phase, and the organic template then regulating the nucleation, growth, orientation, morphology, size, and microstructure of inorganic crystals $(29,30)$.

Experiments show that the surface functional groups of PAMAM dendrimers increase exponentially with the next generation and the concentration of $-\mathrm{NH} 2$ groups may be related to their toxicity (31). The order of cytotoxicity is hydroxyl-terminated $<$ carboxyl-terminated $<$ aminoterminated PAMAM dendrimers (32). Therefore, 

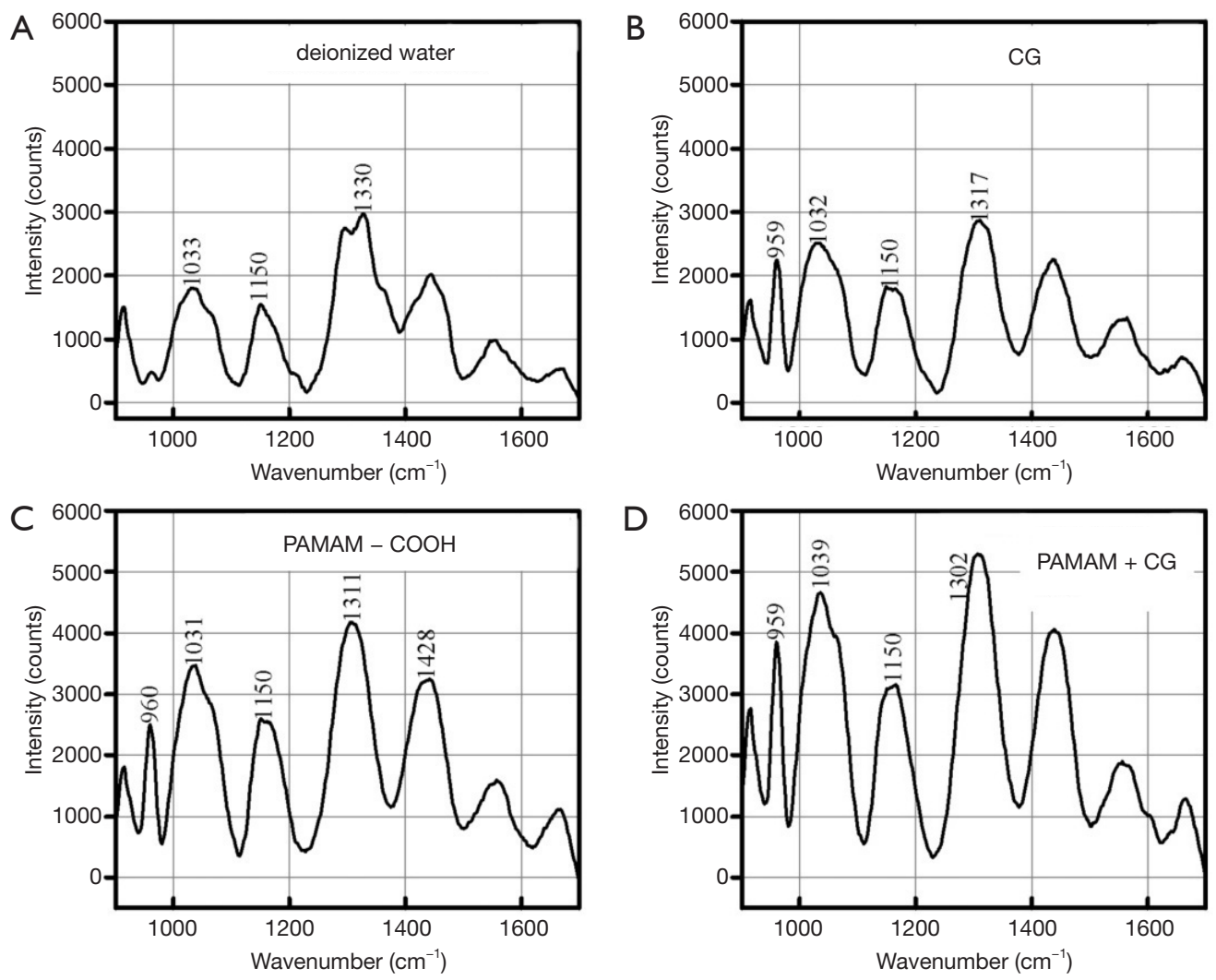

Figure 6 Raman spectroscopic results of dentin after different treatments for 14 days.

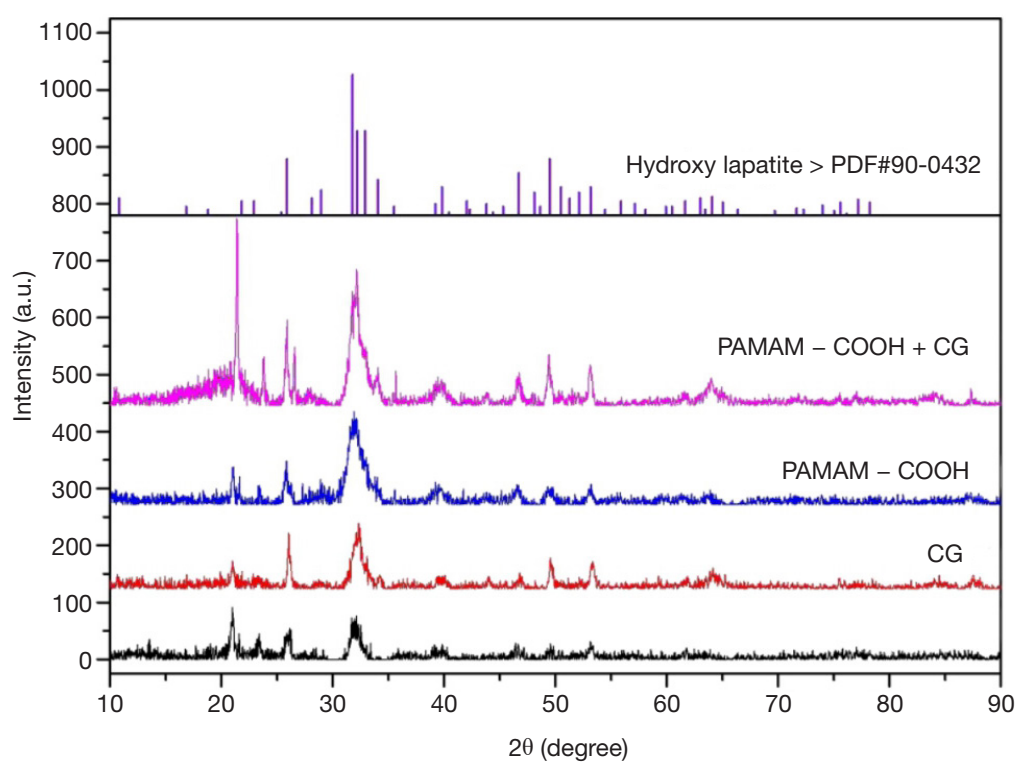

Figure 7 X-ray diffraction (XRD) analysis of dentin after 14 days of different treatments. 
Table 1 XRD diffraction peak intensity and grain size of dentin after different treatments for 14 days

\begin{tabular}{lcccc}
\hline Sample & Grain $(25.8)(\mathrm{nm})$ & Grain (32) $(\mathrm{nm})$ & Aspect ratio (002)/(211) & Peak intensity ratio (002)/(211) \\
\hline Intact dentin & 31.7 & 12 & 10.89 & 0.68 \\
PAMAM & 26 & 7.95 & 3.27 & 1.04 \\
CG & 41.95 & 26.9 & 1.56 & 0.73 \\
PAMAM + CG & 73.45 & 10.85 & 6.77 & 0.74 \\
Deionized water & 25.5 & 30 & 0.85 & 0.7 \\
\hline
\end{tabular}

XRD, X-ray diffraction; PAMAM, polyamide-amine dendrimer; CG, chlorhexidine gluconate.

considering further clinical applications, 4.5 G PAMAM$\mathrm{COOH}$ was used as the research object in this study.

The formation of crystal depends on the ionic product being larger than the solubility product constant of solid phase formed on resin and teeth under experimental conditions. With a gap of $50 \mu \mathrm{m}$, the microenvironment may make the local ion concentration higher than that formed on the external or free surface and exceed the solubility product constants of many mineral forms such as calcium phosphate and calcium fluoride (33).

Through the above experimental results, we found that the dentin treated by PAMAM-COOH formed extensive and obvious peritubular remineralization after 14 days of remineralization in the microenvironment, and the dentin tubes were blocked to varying degrees. The microstructure, growth orientation, and content of the crystal were similar to those of dentin apatite, and the absorption strength of PAMAM molecules for $\mathrm{Ca}$ and $\mathrm{P}$ ions was higher than that of CHX molecules.

After remineralization in the microenvironment for 14 days, the CG group produced visible coverage locally, where mineralized crystals still grew along the $\mathrm{c}$ axis and the average grain size was larger. Although there were some areas where mineralization was not obvious, there were spherical particles gathered in the dentinal canal, proving mineralization occurred. We speculate that the obvious regional differences in CG samples may be related to the slow mineralization rate of CG and the lack of residual crystals on some collagen fibers $(25,34)$. Some studies have shown that there is no significant difference in remineralization effect between $2 \%$ and $0.2 \%$ CG, and the concentration of CG seems to be saturated and if it exceeds this concentration, the remineralization degree will not increase. Therefore, using CG alone to induce dentin remineralization still has some limitations.

After remineralization in the microenvironment for
14 days, the PAMAM-COOH + CG group produced extensive coverage visible to the naked eye. Under electron microscope scanning, a layer of dense lamellar mineralization was seen to have formed on the surface, and the dentinal canal was completely blocked. On the contrary, little sediment adhered to the dentin surface after immersion in artificial saliva for 14 days in the deionized water group, and the dentinal tubules remained open, suggesting that saliva alone could not effectively mineralize and seal the exposed dentinal tubules. The results of EDX surface scanning and the peak intensity of $\mathrm{Ca}$ and $\mathrm{P}$ indicate that the absorption intensity of the complex of PAMAM$\mathrm{COOH}$ and $\mathrm{CG}$ for $\mathrm{Ca}$ and $\mathrm{P}$ ions is higher than that of the other three groups, and the ratio of $\mathrm{Ca} / \mathrm{P}$ in sediments is 1.87 , which is close to hydroxyapatite. At the same time, the relatively high concentration of $\mathrm{C}$ and $\mathrm{O}$ on the surface of the PAMAM group and PAMAM + CG group also confirmed that PAMAM, as an organic macromolecule, has a strong adsorption on the dentin surface. Raman results showed that there were strong phosphate characteristic peaks at $959 \mathrm{~cm}^{-1}$ and $1,031 \mathrm{~cm}^{-1}$, and XRD results showed diffraction peaks like those of hydroxyapatite in the PDF card, which are high and sharp with few burrs, indicating high crystallinity and high purity of the crystal. Calculating the grain size after single peak fitting showed the growth orientation and grain aspect ratio of mineralized crystals were close to those of dentin apatite, and the average grain size in the $\mathrm{C}$ axis direction was larger than that of healthy dentin. The above results suggest that the compound of PAMAM-COOH and CG has a good ability to induce dentin remineralization, and the remineralization structure of dentin surface may be hydroxyapatite crystal.

In conclusion, the combination of polyamide-amine dendrimer and chlorhexidine can induce remineralization of completely demineralized dentin in the resin dentin bonding microenvironment with a gap of $50 \mu \mathrm{m}$, and the 
crystal mineralized layer similar to dentin hydroxyapatite formed on the dentin surface can completely close the exposed dentin tubules. These findings may stimulate further research on the use of composites to play dual roles of remineralization and MMPs inhibition at the resin dentin bonding interface to reduce microleakage, protect dentin collagen, and improve the long-term effect of resin-dentin bonding.

\section{Acknowledgments}

Funding: Youth Fund of National Natural Science Foundation of China (8170095); Chongqing Basic Research and Preface Exploration Project (cstc2018jcyjAX0275).

\section{Footnote}

Reporting Checklist: The authors have completed the MDAR reporting checklist. Available at http://dx.doi.org/10.21037/ atm-21-472

Data Sharing Statement: Available at http://dx.doi. org/10.21037/atm-21-472

Conflicts of Interest: All authors have completed the ICMJE uniform disclosure form (available at http://dx.doi. org/10.21037/atm-21-472). The authors have no conflicts of interest to declare.

Ethical Statement: The authors are accountable for all aspects of the work in ensuring that questions related to the accuracy or integrity of any part of the work are appropriately investigated and resolved. All procedures performed in this study involving human participants were in accordance with the Declaration of Helsinki (as revised in 2013). The study was approved by the Ethics Committee of Stomatological Hospital Affiliated to Chongqing Medical University (No. 2019-75) and informed consent was taken from all the patients.

Open Access Statement: This is an Open Access article distributed in accordance with the Creative Commons Attribution-NonCommercial-NoDerivs 4.0 International License (CC BY-NC-ND 4.0), which permits the noncommercial replication and distribution of the article with the strict proviso that no changes or edits are made and the original work is properly cited (including links to both the formal publication through the relevant DOI and the license).
See: https://creativecommons.org/licenses/by-nc-nd/4.0/.

\section{References}

1. De Munck J, Van Landuyt K, Peumans M, et al. A critical review of the durability of adhesion to tooth tissue: methods and results. J Dent Res 2005;84:118-32.

2. De Munck J, Mine A, Poitevin A, et al. Meta-analytical review of parameters involved in dentin bonding. J Dent Res 2012;91:351-7.

3. Drummond JL. Degradation, fatigue, and failure of resin dental composite materials. J Dent Res 2008;87:710-9.

4. Liang K, Yuan H, Li J, et al. Remineralization of demineralized dentin induced byamine-terminated pamam dendrimer. Macromol Mater Eng 2015;300:107-17. (http://pdf.xuebalib.com:1262/1a871DoHTW5h.pdf)

5. Chen M, Yang J, Li J, et al. Modulated regeneration of acid-etched human tooth enamel by a functionalized dendrimer that is an analog of amelogenin. Acta Biomater 2014;10:4437-46.

6. Kuboki Y, Ohgushi K, Fusayama T. Collagen biochemistry of the two layers of carious dentin. J Dent Res 1977;56:1233-7.

7. González-Cabezas C. The chemistry of caries: remineralization and demineralization events with direct clinical relevance. Dent Clin North Am 2010;54:469-78.

8. Xu Z, Neoh KG, Kishen A. A biomimetic strategy to form calcium phosphate crystals on type i collagen substrate. Mater Sci Eng C 2010;30:822-6. (https://www. researchgate.net/publication/223673561_A_biomimetic_ strategy_to_form_calcium_phosphate_crystals_on_type_I_ collagen_substrate)

9. Peters MC. Strategies for noninvasive demineralized tissue repair. Dent Clin North Am 2010;54:507-25.

10. Sauro S, Osorio R, Watson TF, et al. Influence of phosphoproteins' biomimetic analogs on remineralization of mineral-depleted resin-dentin interfaces created with ion-releasing resin-based systems. Dent Mater 2015;31:759-77.

11. Osorio R, Osorio E, Medina-Castillo AL, et al. Polymer nanocarriers for dentin adhesion. J Dent Res 2014;93:1258-63.

12. Sabatini C, Scheffel DL, Scheffel RH, et al. Inhibition of endogenous human dentin MMPs by Gluma. Dent Mater 2014;30:752-8.

13. Khaddam M, Salmon B, Le Denmat D, et al. Grape seed extracts inhibit dentin matrix degradation by MMP-3. Front Physiol 2014;5:425. 
14. Dickens SH, Flaim GM, Takagi S. Mechanical properties and biochemical activity of remineralizing resin-based $\mathrm{Ca}$ PO4 cements. Dent Mater 2003;19:558-66.

15. Liang K, Weir MD, Xie X, et al. Dentin remineralization in acid challenge environment via PAMAM and calcium phosphate composite. Dent Mater 2016;32:1429-40.

16. Melo MA, Orrego S, Weir MD, et al. Designing Multiagent Dental Materials for Enhanced Resistance to Biofilm Damage at the Bonded Interface. ACS Appl Mater Interfaces 2016;8:11779-87.

17. Gendron R, Grenier D, Sorsa T, et al. Inhibition of the activities of matrix metalloproteinases 2, 8, and 9 by chlorhexidine. Clin Diagn Lab Immunol 1999;6:437-9.

18. Carrilho MR, Carvalho RM, Sousa EN, et al. Substantivity of chlorhexidine to human dentin. Dent Mater 2010;26:779-85.

19. de Amorim RG, Leal SC, Bezerra AC, et al. Association of chlorhexidine and fluoride for plaque control and white spot lesion remineralization in primary dentition. Int J Paediatr Dent 2008;18:446-51.

20. Kim DS, Kwon JM, Park SH, et al. Mechanical and micromorphological evaluation of chlorhexidine-mediated dentin remineralization. Scanning 2012;34:151-8.

21. Ricci HA, Sanabe ME, de Souza Costa CA, et al. Chlorhexidine increases the longevity of in vivo resindentin bonds. Eur J Oral Sci 2010;118:411-6.

22. Gao Y, Liang K, Li J, et al. Effect and Stability of Poly(Amido Amine)-Induced Biomineralization on Dentinal Tubule Occlusion. Materials (Basel) 2017;10:384.

23. Chen L, Liang K, Li J, et al. Regeneration of biomimetic hydroxyapatite on etched human enamel by anionic PAMAM template in vitro. Arch Oral Biol 2013;58:975-80.

24. Liang K, Gao Y, Li J, et al. Effective dentinal tubule occlusion induced by polyhydroxy- terminated pamam dendrimer in vitro. Rsc Adv 2014;4:43496-503. (http://

Cite this article as: Xiang K, Chen L, Chen W, Yang D. Remineralization of dentin induced by a compound of polyamide-amine and chlorhexidine in a resin dentin bonding microenvironment. Ann Transl Med 2021;9(6):472. doi: 10.21037/atm-21-472
pdf-s3.xuebalib.com:1262/1a88Ph1AiSqn.pdf)

25. Chen L, Yuan H, Tang B, et al. Biomimetic remineralization of human enamel in the presence of polyamidoamine dendrimers in vitro. Caries Res 2015;49:282-90.

26. Tay FR, Pashley DH. Guided tissue remineralisation of partially demineralised human dentine. Biomaterials 2008;29:1127-37. (https://www.sciencedirect.com/science/ article/abs/pii/S0142961207008940?via\%3Dihub)

27. Luo JJ, Ning TY, Li QL, et al. A model for biomimetic mineralization of enamel. Oral Biomedicine 2016;7:173-6.

28. Li J, Yang J, Li J, et al. Bioinspired intrafibrillar mineralization of human dentine by PAMAM dendrimer. Biomaterials 2013;34:6738-47.

29. Mai S, Kim YK, Kim J, et al. In vitro remineralization of severely compromised bonded dentin. J Dent Res 2010;89:405-10.

30. Mai S, Kim YK, Toledano M, et al. Phosphoric acid esters cannot replace polyvinylphosphonic acid as phosphoprotein analogs in biomimetic remineralization of resin-bonded dentin. Dent Mater 2009;25:1230-9.

31. Gao F, Djordjevic I, Pokholenko O, et al. On-Demand Bioadhesive Dendrimers with Reduced Cytotoxicity. Molecules 2018;23:796.

32. El-Sayed M, Ginski M, Rhodes C, et al. Transepithelial transport of poly(amidoamine) dendrimers across Caco-2 cell monolayers. J Control Release 2002;81:355-65.

33. Hashimoto M, Nakamura K, Kaga M, et al. Crystal growth by fluoridated adhesive resins. Dent Mater 2008;24:457-63.

34. Sear RP. The non-classical nucleation of crystals: Microscopic mechanisms and applications to molecular crystals, ice and calcium carbonate. Int. Mater. Rev 2013;57:328-56.

(English Language Editor: B. Draper) 